\title{
Pre-diagnostic meat and fibre intakes in relation to colorectal cancer survival in the European Prospective Investigation into Cancer and Nutrition
}

Heather A. Ward ${ }^{1}$, Teresa Norat ${ }^{1}$, Kim Overvad $^{2}$, Christina C. Dahm ${ }^{2}$, H. Bas Bueno-de-Mesquita ${ }^{1,3,4,5}$, Mazda Jenab $^{6}$, Veronika Fedirko ${ }^{7}$, Fränzel J. B. van Duijnhoven ${ }^{8,9}$, Guri Skeie ${ }^{10}$, Dora Romaguera-Bosch ${ }^{1,11,12}$, Anne Tjønneland ${ }^{13}$, Anja Olsen ${ }^{13}$, Franck Carbonnel ${ }^{14,15,16}$, Aurélie Affret ${ }^{14,15,16}$, Marie-Christine Boutron-Ruault ${ }^{14,15,16}$, Verena Katzke ${ }^{17}$, Tilman Kühn ${ }^{17}$, Krassimira Aleksandrova $^{18}$, Heiner Boeing ${ }^{18}$, Antonia Trichopoulou ${ }^{19,20}$, Pagona Lagiou ${ }^{19,20,21}$, Christina Bamia ${ }^{19,20}$, Domenico Palli ${ }^{22}$, Sabina Sieri ${ }^{23}$, Rosario Tumino ${ }^{24}$, Alessio Naccarati ${ }^{25}$, Amalia Mattiello $^{26}$, Petra H. Peeters ${ }^{1,27}$, Elisabete Weiderpass ${ }^{28,29,30,31}$, Lene Angell Åsli ${ }^{28}$, Paula Jakszyn $^{32}$, J. Ramón Quirós ${ }^{33}$, María-José Sánchez ${ }^{34,35}$, Miren Dorronsoro ${ }^{36}$, José-María Huerta ${ }^{35,37}$, Aurelio Barricarte ${ }^{35,38,39}$, Karin Jirström ${ }^{40}$, Ulrika Ericson ${ }^{41}$, Ingegerd Johansson ${ }^{42}$, Björn Gylling ${ }^{43}$, Kathryn E. Bradbury ${ }^{44}$, Kay-Tee Khaw ${ }^{45}$, Nicholas J. Wareham ${ }^{46}$, Magdalena Stepien ${ }^{6}$, Heinz Freisling ${ }^{6}$, Neil Murphy ${ }^{1}$, Amanda J. Cross ${ }^{1}$ and Elio Riboli ${ }^{1}$

${ }^{1}$ Imperial College London, London W2 $1 P G$, UK

${ }^{2}$ Section for Epidemiology, Department of Public Health, Aarhus University, Bartholins Allé 2 - Building 1260 , DK-8000 Aarbus C, Denmark

${ }^{3}$ Department for Determinants of Chronic Diseases (DCD), National Institute for Public Health and the Environment (RIVM), Antonie van Leeuwenhoeklaan 9, PO Box 1,3720 BA Bilthoven, The Netherlands

${ }^{4}$ Department of Gastroenterology and Hepatology, University Medical Center, PO 85500, 3508, GA, Utrecht, The Netherlands ${ }^{5}$ Department of Social \& Preventive Medicine, Faculty of Medicine, University of Malaya, 50603 Kuala Lumpur, Malaysia

${ }^{6}$ International Agency for Research on Cancer (WHO-IARC), 150 Cours Albert Thomas, 69372 Lyon CEDEX 08, France

${ }^{7}$ Department of Epidemiology, Rollins School of Public Health, Emory University, 201 Dowman Drive, Atlanta, GA 30322, USA

${ }^{8}$ Division of Human Nutrition (Bode 62), PO Box 8129, 6700 EV Wageningen, The Netherlands

${ }^{9}$ National Institute for Public Health and the Environment, PO Box 1, 3720 BA Bilthoven, The Netherlands

${ }^{10}$ UiT The Arctic University of Norway, Postboks 6050, Langnes, 9037 Tromsø, Norway

${ }^{11}$ Instituto de Investigación Sanitaria de Palma (IdISPa), Carretera de Valldemossa, 79, Hospital Universitario Son Espases, Edificio S, O7120 Palma de Mallorca, Illes Balears, Spain

${ }^{12}$ CIBER Fisiopatología de la Obesidad y Nutrición (CIBER-OBN), Instituto de Salud Carlos III, C/ Monforte de Lemos 3-5, Pabellón 11, Planta O, 28029 Madrid, Spain

${ }^{13}$ Danish Cancer Society Research Center, Strandboulevarden 49, DK-2100 København Ø, Denmark

${ }^{14}$ Center for Research in Epidemiology and Population Health (CESP), U1018, Lifestyle, Genes and Health: Integrative

Trans-Generational Epidemiology, Institut National de la Santé et de la Recherche Médicale, F-94805 Villejuif, France

${ }^{15}$ Université Paris Sud, UMRS 1018, F-94805 Villejuif, France

${ }^{16}$ Gustave Roussy, F-94805 Villejuif, France

${ }^{17}$ German Cancer Research Center (DKFZ), Division of Cancer Epidemiology, Im Neuenheimer Feld 280, 69120 Heidelberg, Germany

${ }^{18}$ Department of Epidemiology, German Institute of Human Nutrition (DIfE), Arthur-Scheunert-Allee 114-116, 14558

Bergholz-Rehbrücke, Germany

${ }^{19}$ Hellenic Health Foundation, Kaisareias 13 \& Alexandroupoleos, GR-115 27, Athens, Greece

${ }^{20}$ Department of Hygiene, Epidemiology and Medical Statistics, WHO Collaborating Center for Nutrition and Health,

Unit of Nutritional Epidemiology and Nutrition in Public Health, University of Athens Medical School, Mikras Asias 75, Goudi, GR-11527, Athens, Greece

${ }^{21}$ Department of Epidemiology, Harvard School of Public Health, 677 Huntington Avenue, Boston, MA O2115, USA

${ }^{22}$ ISPO - Cancer Research and Prevention Institute, Clinical and Descriptive Epidemiology Unit, Via delle Oblate 2, 50141, Florence, Italy

Abbreviations: CRC, colorectal cancer; EPIC, European Prospective Investigation into Cancer and Nutrition; HR, hazard ratio; SSB, sugar-sweetened beverages.

* Corresponding author: H. A. Ward, email Heather.ward@imperial.ac.uk 
${ }^{23}$ Epidemiology and Prevention Unit, Fondazione IRCCS Istituto Nazionale dei Tumouri, Via Giacomo Venezian 1, 20133 Milan, Italy

${ }^{24}$ Cancer Registry and Histopathology Unit, 'Civic-M.P.Arezzo' Hospital, ASP, Via Dante No. 109, Ragusa 97100, Italy

${ }^{25}$ Molecular and Genetic Epidemiology Unit, Human Genetics Foundation (HuGeF), Via Nizza 52, 10126 Torino, Italy

${ }^{26}$ Dipartimento di Medicina Clinica e Chirurgia, Federico II University, Via Pansini, 5-80131 - Naples, Italy

${ }^{27}$ Department of Epidemiology, Julius Center for Health Sciences and Primary Care, University Medical Center Utrecht, Huispost Str. 6.131, PO Box 85500, 3508 GA, Utrecht, The Netherlands

${ }^{28}$ Department of Community Medicine, Faculty of Health Sciences, University of Tromsø - The Arctic University of Norway, Postboks 6050 Langnes, 9037 Tromsø, Norway

${ }^{29}$ Department of Research, Cancer Registry of Norway, Institute of Population-Based Cancer Research, PO Box 5313 Majorstuen, N-O304 Oslo, Norway

${ }^{30}$ Department of Medical Epidemiology and Biostatistics, Karolinska Institute, PO Box 281, SE-171 77 Stockholm, Sweden

${ }^{31}$ Genetic Epidemiology Group, Folkhälsan Research Center, FI-O0290 Helsinki, Finland

${ }^{32}$ Unit of Nutrition, Environment and Cancer, Catalan Institute of Oncology, Avda Gran Via 199-203, 08908 L'Hospitalet de Llobregat, Barcelona, Spain

${ }^{33}$ Public Health Directorate, Asturias, Ciriaco Miguel Vigil St 9, Oviedo 33006, Spain

${ }^{34}$ Escuela Andaluza de Salud Pública, Instituto de Investigación Biosanitaria ibs, Hospitales Universitarios de Granada/ Universidad de Granada, Cuesta del Observatorio, 4, Campus Universitario de Cartuja, Granada 18080, Spain

${ }^{35}$ CIBER de Epidemiología y Salud Pública (CIBERESP), Melchor Fernández Almagro, 3-5, Madrid 28029, Spain

${ }^{36}$ Basque Regional Health Department, Public Health Direction and Biodonostia Research Institute - CIBERESP, Avenida de Navarra, 4, 20013 Donostia-San Sebastián, Spain

${ }^{37}$ Department of Epidemiology, Murcia Regional Health Council, IMIB-Arrixaca, Ronda de Levante 11, Murcia 30008, Spain ${ }^{38}$ Navarra Public Health Institute, c/Leyre 15, Pamplona 31003, Spain

${ }^{39}$ Navarra Institute for Health Research, Recinto de Complejo Hospitalario de Navarra c/Irunlarrea 3, Pamplona 31008, Spain ${ }^{40}$ Department of Clinical Sciences, Division of Oncology and Pathology, Lund University, Box 117, SE-221 OO Lund, Sweden ${ }^{41}$ Diabetes and Cardiovascular Disease - Genetic Epidemiology, Department of Clinical Sciences, Lund University, Box 117, SE-221 OO Lund, Sweden

${ }^{42}$ Department of Odontology, Umeå University, SE-901 87 Umeå, Sweden

${ }^{43}$ Department of Pathology, Umeå University, SE-901 87 Umeå, Sweden

${ }^{44}$ Cancer Epidemiology Unit, Nuffield Department of Population Health, University of Oxford, Richard Doll Building, Oxford OX3 7LF, UK

${ }^{45}$ Department of Public Health and Primary Care, University of Cambridge, Addenbrooke's Hospital, Hills Rd, Cambridge CB2 OSP, UK

${ }^{46}$ Medical Research Council Epidemiology Unit, University of Cambridge, Institute of Metabolic Science, Box 285, Addenbrooke's Hospital, Hills Road, Cambridge CB2 OQQ, UK

(Submitted 26 January 2016 - Final revision received 15 March 2016 - Accepted 11 April 2016 - First published online 19 May 2016)

\section{Abstract}

Improvements in colorectal cancer (CRC) detection and treatment have led to greater numbers of CRC survivors, for whom there is limited evidence on which to provide dietary guidelines to improve survival outcomes. Higher intake of red and processed meat and lower intake of fibre are associated with greater risk of developing CRC, but there is limited evidence regarding associations with survival after CRC diagnosis. Among 3789 CRC cases in the European Prospective Investigation into Cancer and Nutrition (EPIC) cohort, pre-diagnostic consumption of red meat, processed meat, poultry and dietary fibre was examined in relation to CRC-specific mortality ( $n$ 1008) and all-cause mortality $(n 1262)$ using multivariable Cox regression models, adjusted for CRC risk factors. Pre-diagnostic red meat, processed meat or fibre intakes (defined as quartiles and continuous grams per day) were not associated with CRC-specific or all-cause mortality among CRC survivors; however, a marginal trend across quartiles of processed meat in relation to CRC mortality was detected $(P$ 0.053). Pre-diagnostic poultry intake was inversely associated with all-cause mortality among women (hazard ratio (HR)/20 g/d 0.92; 95\% CI 0.84, 1.00), but not among men (HR 1.00; $95 \%$ CI 0.91, 1.09) ( $P_{\text {for heterogeneity }}=0 \cdot 10$ ). Pre-diagnostic intake of red meat or fibre is not associated with CRC survival in the EPIC cohort. There is suggestive evidence of an association between poultry intake and all-cause mortality among female CRC survivors and between processed meat intake and CRC-specific mortality; however, further research using post-diagnostic dietary data is required to confirm this relationship.

Key words: Colorectal cancers: Cancer survival: Diets: Cohorts: European Prospective Investigation into Cancer and Nutrition 
Diet is thought to play a key role in cancer prevention, with more than half of colorectal cancers (CRC) potentially preventable through diet and lifestyle modifications ${ }^{(1)}$. However, the role of diet on CRC survival is unclear. With an estimated 244000 CRC patients in the UK, this is an urgent area of research. There are a number of plausible mechanisms to link diet to CRC, including formation of $N$-nitroso compounds ${ }^{(2)}$, anti-inflammatory effects $^{(3)}$, contributions to DNA synthesis and methylation ${ }^{(4)}$, inhibition of cancer cell proliferation ${ }^{(5,6)}$, binding to free fatty acids and bile acids ${ }^{(7)}$, production of anticancer SCFA ${ }^{(8)}$, insulin lowering $^{(9)}$ and metabolic activity attributed to adiposity ${ }^{(10)}$. In particular, convincing evidence has been presented for foods containing dietary fibre (lower CRC risk) and for red and processed meat (higher CRC risk) ${ }^{(11)}$; these pathways may also be relevant for CRC recurrence and survival outcomes. CRC patients frequently request dietary recommendations ${ }^{(12)}$, but there is insufficient evidence at present to provide guidance ${ }^{(13)}$. This is an increasingly necessary area of research as CRC survival rates have improved because of earlier detection and advances in treatment in many Western countries ${ }^{(14,15)}$; between 1989 and 2011, mortality has decreased among European CRC patients by $13 \%$ in men and $27 \%$ in women ${ }^{(16)}$.

At present, the few available studies on CRC survival and consumption of meat and fibre have yielded inconclusive findings. In the Cancer Prevention Study II, post-diagnostic intake of red and processed meat was not associated with survival among CRC patients ${ }^{(17)}$. However, pre-diagnostic intake of red and processed meat was associated with greater all-cause mortality, and those with high intake of red and processed meat during both pre- and post-diagnostic dietary assessments were at greater risk of CRC-specific mortality ${ }^{(17)}$. In a smaller US study, post-diagnosis red meat intake was associated with higher mortality risk, but only among those with familial $\mathrm{CRC}^{(18)}$. Results for post-diagnostic fibre intake and CRC survival are similarly mixed. Among Mormons in Utah (USA), higher fibre intake was associated with poorer mortality outcomes ${ }^{(19)}$, whereas another US study ${ }^{(18)}$ yielded null results. Pre-diagnostic fibre intake was also unrelated to survival outcomes among CRC patients in studies from France ${ }^{(20)}$ and Scandinavia ${ }^{(21)}$. However, many of the aforementioned studies had relatively small sample sizes $\left(<500\right.$ CRC cases $\left.{ }^{(18,19,21)}\right)$ and may have been underpowered to detect statistically significant associations. Drawing on the large European Prospective Investigation into Cancer and Nutrition (EPIC) cohort, the objective of the present analysis was to further characterise the role of pre-diagnostic meat and fibre intakes on survival among CRC cases, with separate consideration for red meat, processed meat and poultry.

\section{Methods \\ Study population}

EPIC is a multi-centre, prospective cohort study, which recruited 519978 volunteers (mostly ages 25-70 years) from twentythree centres in ten countries (Sweden, Denmark, Norway, The Netherlands, UK, France, Germany, Spain, Italy and Greece) between 1992 and 2000. The study has been described in detail previously ${ }^{(22,23)}$. EPIC was approved by the Institutional Review Board at the International Agency for Research on Cancer (Lyon, France) and local ethics committees, and informed consent was obtained from all participants.

\section{Data collection and definitions}

At baseline, participants reported dietary intake using countryspecific validated questionnaires. In most centres, a selfadministered FFQ was used to assess intake over the past 12 months (eighty-eight to 266 food items). A questionnaire plus food record was used in Malmö, Sweden, and intervieweradministered diet questionnaires were used in Greece, Spain and Ragusa, Italy. For this analysis, red meat included beef, pork, mutton/lamb, horse, goat; processed meat included all meat products including ham, bacon, sausages and a small part of minced meat in ready-to-eat products; and poultry included chicken, hen, turkey, duck, goose, unclassified poultry and rabbit (domestic). Dietary intake of fibre was calculated from the standardised EPIC Nutrient Data Base ${ }^{(24)}$. Questionnaires on education, occupation, previous illnesses, alcohol and tobacco consumption, and physical activity were also completed by participants. Anthropometric variables were measured in most centres; as exceptions, self-reported data were used in France and Norway, and in Oxford self-reported data were adjusted based on measurements collected from a subset of participants ${ }^{(25)}$.

Population cancer registries were used in Denmark, Italy, The Netherlands, Norway, Spain, Sweden and the UK to identify incident cancers. In France, Germany and Greece, cancer cases were identified through active follow-up, directly through study participants or next of kin, and confirmed by a combination of methods including health insurance records as well as cancer and pathology registries. CRC cases were identified according to the second edition of the International Classification of Diseases for Oncology (ICD-O), either as located in the colon (C18.0-C18.7), rectum (C19 and C20) or overlapping/unspecified (C18.8 and 18.9).

The date of CRC diagnosis was used as the start date of followup for the present study. Participants were censored at date of death, last date of contact or the date at which follow-up data were considered to be complete at each study centre (between June 2005 and June 2009). Loss to follow-up across all countries was low $(<2 \%)$. Harmonisation of tumour stage data was required to combine TNM (Tumour, Node, Metastasis) classification classification, Dukes's classification or centre-specific classification; this process has been described previously ${ }^{(26)}$.

A total of 4701 CRC cases were identified. Cases excluded from the present analysis were as follows: 426 cases diagnosed with CRC after vital status censoring date, 172 cases with in situ or metastatic tumour, 144 non-adenocarcinoma or tumour of unknown morphology, twenty-one because of missing date of death or diagnosis, eight missing cause of death, seventy-four within the extreme ranking (top and bottom $1 \%$ ) of the ratio energy intake:energy requirement and sixty-seven with missing data on diet. The final sample included 3789 CRC cases (1603 men and 2186 women; 2383 colon cancer cases and 1406 rectal cancer cases). 
Availability of data on tumour stage and grade varied between centres: there were 970 participants with missing stage data, including all participants from Malmö ( $n$ 353) and Oxford ( $n$ 271), and there were 2080 participants with missing grade data, including all participants from Denmark ( $n$ 705), Malmö ( $n$ 353), Cambridge ( $n$ 270) and Oxford ( $n$ 271), as well as over $85 \%$ of participants from Umeå ( $n$ 155).

Mortality data were obtained at the regional or national level. In Denmark, Italy, The Netherlands, Norway, Spain, Sweden and the UK, vital status and the causes and dates of death were ascertained by death indices, cancer registry records and national health statistics. Active follow-up was adopted in Germany, Greece and France. Causes of death were coded according to the International Classification of Diseases, 10th Revision (ICD-10). Up to six qualifiers of the cause of death were reviewed. Death from CRC was assigned based on the underlying cause of death.

\section{Statistical analysis}

The primary end point in the present analysis was death due to CRC (with other causes of death modelled as competing risks, using SAS macro PSHREG ${ }^{(27)}$ ); the secondary end point was allcause mortality. The pre-diagnostic dietary exposures in the present analysis, rescaled to reflect approximately $1 \mathrm{sD}$, were fibre $(10 \mathrm{~g} / \mathrm{d})$, red meat $(40 \mathrm{~g} / \mathrm{d})$, processed meat $(30 \mathrm{~g} / \mathrm{d})$, combined red and processed meat $(50 \mathrm{~g} / \mathrm{d})$ and poultry $(20 \mathrm{~g} / \mathrm{d})$; separate models were built for each dietary exposure. Sex-specific quartiles (Q) of the dietary variables were also derived for analysis. Fibre was further analysed by source (cereal, fruit and vegetable) as quartile and continuous grams per day. Tests for trend across quartiles were conducted by assigning participants the sex-specific median value per quartile.

The proportional hazards models used age at CRC diagnosis and age at censoring as the underlying time variables, and the models were stratified by country; two models are presented: (i) a model that adjusts for age at diagnosis and sex and (ii) a multivariable model adjusting for additional confounders. Potentially confounding variables were selected on the basis of possible associations with both the outcome and the dietary exposures, and were entered into the model stepwise to determine whether they changed the association between dietary exposure and mortality by $10 \%$ or more. The following variables were identified for one or more of the dietary exposures under study: education level (primary school, technical/professional school, secondary school, longer education (including university degree), not specified/missing), smoking status (current, former, never, unknown), BMI ( $\mathrm{kg} / \mathrm{m}^{2}$, continuous), total energy intake $(\mathrm{kJ} / \mathrm{d}(\mathrm{kcal} / \mathrm{d}))$, Ca intake $(\mathrm{mg} / \mathrm{d})$, folate intake $(\mathrm{mg} / \mathrm{d})$, alcohol intake $(\mathrm{g} / \mathrm{d}$ : $0,>0-6(\mathrm{M}) />0-3(\mathrm{~W}),>6-12(\mathrm{M}) />3-12(\mathrm{~W})$, $>12-24,>24-60,>60$ ), tumour grade (well differentiated, moderately differentiated, poorly/undifferentiated, unknown), tumour stage (localised (I), localised with invasion (II), metastatic regional (III), metastatic distant (IV), unknown) and year of diagnosis (continuous, calendar year). Adjustment for physical activity (the Cambridge Index categories ${ }^{(28)}$ ) and cancer site (colon $v$. rectum) did not change any associations by $10 \%$ or more, and were therefore not included. The independent effect of red meat, processed meat, poultry and fibre was explored by mutually adjusting for each other in an additional model. The validity of the proportional hazards assumption was tested by inclusion of time-varying covariates; there was no evidence of deviation from proportionality.

Potential interactions with pre-diagnostic red meat, poultry, processed meat and fibre as continuous variables were tested for education level (primary school, technical/professional/secondary school, longer education) smoking status (current, former, never), BMI $\left(<25,25-30,>30 \mathrm{~kg} / \mathrm{m}^{2}\right)$, intake of total energy $(\mathrm{kJ} / \mathrm{d}(\mathrm{kcal} / \mathrm{d}))$, Ca intake $(\mathrm{mg} / \mathrm{d})$, folate intake $(\mathrm{mg} / \mathrm{d})$, alcohol intake (g/d: 0, >0-6 (M)/>0-3 (W), >6-24 (M)/>3-24 (W), $\geq 24$ ), stage (I-III $v$. IV), age at diagnosis $(<60,60-69, \geq 70$ years), year of diagnosis (1992-1999, 2000-2004, 2005-2009), median length of follow-up after CRC diagnosis $(<3 \cdot 3 v$. $\geq 3 \cdot 3$ years) and median length of time between dietary assessment and CRC diagnosis $(<6.5 v . \geq 6.5$ years). The significance of interaction terms was assessed by likelihood ratio tests comparing nested models with and without the interaction terms.

A number of sensitivity analyses were conducted. Waist circumference was identified as a potential confounder, but was examined in a supplementary model rather than the main analysis because of a relatively high proportion of missing data ( $n$ 566). In order to explore the potential influence of advanced $\mathrm{CRC}$, the main models were reanalysed (i) among participants with stage I, II or III tumours and (ii) restricted to deaths occurring more than 6 months after recruitment. The effect of replacing red and processed meat with poultry was estimated by substitution models, where total intake of red meat, processed meat and poultry was held constant and an increase in poultry intake represented an equal decrease in red and processed meat intake ${ }^{(29)}$.

Tumour stage data were missing for $25.6 \%$ of participants. The influence of missing stage data was examined by three ways: (i) creation of a separate 'missing' category for Malmö and Oxford, (ii) exclusion of Oxford and Malmö and (iii) imputation of missing values using SAS imputation command PROC MI. Stage was assumed to be missing at random, and imputation was based on sex, age at CRC diagnosis, year of diagnosis, tumour site, period between CRC diagnosis and death or censoring and vital status as covariates (five iterations).

\section{Results}

Among 1603 men and 2186 women, there were 1008 deaths due to CRC (1262 deaths from all causes). The average length of follow-up since CRC diagnosis was $4 \cdot 1$ years. Baseline characteristics according to low (Q1) and high (Q4) prediagnostic intakes of red and processed meat, poultry and fibre are presented separately for men and women in Table 1. For men and women, those in the highest quartile of red and processed meat intake had relatively higher waist circumference measurements and included a larger proportion of smokers and those with lower levels of education. In contrast, those in the highest quartile of poultry intake had lower waist circumference values than those in Q1. Among men, there was a higher proportion of rectal cancers in Q4 for red and 
Table 1. Demographic, anthropometric and lifestyle characteristics according to pre-diagnostic meat and fibre intakes among colorectal cancer cases in European Prospective Investigation into Cancer and Nutrition

\begin{tabular}{|c|c|c|c|c|c|c|c|c|c|c|c|c|}
\hline & \multicolumn{6}{|c|}{ M } & \multicolumn{6}{|c|}{ W } \\
\hline & \multicolumn{2}{|c|}{ Red and processed meat } & \multicolumn{2}{|c|}{ Fibre } & \multicolumn{2}{|c|}{ Poultry } & \multicolumn{2}{|c|}{ Red and processed meat } & \multicolumn{2}{|c|}{ Fibre } & \multicolumn{2}{|c|}{ Poultry } \\
\hline & Q1 & Q4 & Q1 & Q4 & Q1 & Q4 & Q1 & Q4 & Q1 & Q4 & Q1 & Q4 \\
\hline Age at recruitment (mean) & $59 \cdot 6$ & $56 \cdot 9$ & $58 \cdot 6$ & $57 \cdot 7$ & $59 \cdot 1$ & $58 \cdot 7$ & $58 \cdot 3$ & $56 \cdot 7$ & $57 \cdot 3$ & $57 \cdot 3$ & $57 \cdot 9$ & $57 \cdot 0$ \\
\hline $\mathrm{BMI}$ (mean, $\mathrm{kg} / \mathrm{m}^{2}$ ) & $26 \cdot 7$ & $27 \cdot 7$ & $27 \cdot 2$ & $27 \cdot 1$ & $27 \cdot 1$ & $25 \cdot 1$ & $25 \cdot 1$ & $26 \cdot 3$ & $25 \cdot 9$ & $25 \cdot 5$ & $27 \cdot 7$ & $26 \cdot 5$ \\
\hline Waist circumference (mean) & $95 \cdot 9$ & $98 \cdot 8$ & $97 \cdot 5$ & $97 \cdot 2$ & $97 \cdot 4$ & $81 \cdot 2$ & $80 \cdot 8$ & $85 \cdot 0$ & 83.0 & $82 \cdot 7$ & $98 \cdot 8$ & $85 \cdot 1$ \\
\hline Energy (mean, kJ/d) & 8590 & 11715 & 7895 & 11937 & 9456 & 10560 & 7121 & 9100 & 6309 & 9782 & 7602 & 8703 \\
\hline Energy (mean, kcal/d) & 2053 & 2800 & 1887 & 2853 & 2260 & 2524 & 1702 & 2175 & 1508 & 2338 & 1817 & 2080 \\
\hline \multicolumn{13}{|l|}{ Smokers (\%) } \\
\hline Never & $30 \cdot 7$ & $17 \cdot 3$ & $24 \cdot 0$ & $27 \cdot 7$ & $26 \cdot 0$ & $27 \cdot 4$ & 59.9 & $51 \cdot 3$ & $47 \cdot 3$ & $56 \cdot 2$ & $51 \cdot 1$ & $58 \cdot 4$ \\
\hline Former & $47 \cdot 6$ & $46 \cdot 6$ & $45 \cdot 2$ & 43.7 & $49 \cdot 3$ & $42 \cdot 5$ & $26 \cdot 9$ & $22 \cdot 6$ & 24.9 & $25 \cdot 5$ & $28 \cdot 3$ & $21 \cdot 1$ \\
\hline Smoker & $18 \cdot 1$ & $35 \cdot 1$ & $29 \cdot 3$ & $26 \cdot 7$ & $24 \cdot 0$ & 27.9 & 11.9 & $24 \cdot 6$ & $26 \cdot 9$ & $17 \cdot 1$ & $19 \cdot 1$ & $18 \cdot 9$ \\
\hline Unknown & 3.5 & 1.0 & 1.5 & $2 \cdot 0$ & 0.8 & $2 \cdot 2$ & $1 \cdot 3$ & 1.5 & 0.9 & $1 \cdot 1$ & 1.5 & 1.6 \\
\hline \multicolumn{13}{|l|}{ Alcohol $(\%, g / d)$} \\
\hline Non-drinker & $12 \cdot 6$ & $4 \cdot 5$ & $8 \cdot 1$ & $7 \cdot 4$ & $9 \cdot 8$ & $8 \cdot 0$ & $17 \cdot 7$ & $13 \cdot 3$ & $19 \cdot 8$ & $13 \cdot 8$ & $15 \cdot 4$ & $17 \cdot 8$ \\
\hline$>0-6(\mathrm{M}) />0-3(\mathrm{~W})$ & 30.5 & $11 \cdot 8$ & $20 \cdot 5$ & $22 \cdot 8$ & $26 \cdot 0$ & $15 \cdot 9$ & $38 \cdot 8$ & $28 \cdot 3$ & $30 \cdot 7$ & 35.6 & 34.9 & $26 \cdot 5$ \\
\hline$>6-12(\mathrm{M}) />3-12(\mathrm{~W})$ & $18 \cdot 1$ & $13 \cdot 5$ & $15 \cdot 2$ & $17 \cdot 5$ & $15 \cdot 3$ & $12 \cdot 9$ & $24 \cdot 6$ & $29 \cdot 6$ & $22 \cdot 8$ & 29.4 & $24 \cdot 0$ & $27 \cdot 4$ \\
\hline$>12-24$ & $14 \cdot 6$ & $20 \cdot 3$ & $16 \cdot 9$ & $17 \cdot 8$ & $19 \cdot 0$ & $18 \cdot 4$ & $11 \cdot 8$ & $15 \cdot 3$ & $15 \cdot 0$ & $10 \cdot 6$ & $14 \cdot 9$ & $16 \cdot 2$ \\
\hline$>24-60$ & $21 \cdot 7$ & $36 \cdot 3$ & $25 \cdot 8$ & $28 \cdot 9$ & $21 \cdot 8$ & $35 \cdot 8$ & $7 \cdot 1$ & $12 \cdot 6$ & $10 \cdot 1$ & $10 \cdot 1$ & $9 \cdot 9$ & $11 \cdot 1$ \\
\hline$>60$ & 2.5 & $13 \cdot 6$ & $13 \cdot 6$ & $5 \cdot 6$ & $8 \cdot 3$ & $9 \cdot 0$ & 0.0 & 0.9 & $1 \cdot 7$ & 0.6 & 0.9 & $1 \cdot 1$ \\
\hline \multicolumn{13}{|l|}{ Education (\%) } \\
\hline Primary school & $37 \cdot 8$ & $43 \cdot 1$ & $46 \cdot 0$ & $38 \cdot 3$ & $42 \cdot 0$ & $45 \cdot 5$ & $28 \cdot 0$ & $35 \cdot 2$ & $37 \cdot 8$ & $29 \cdot 1$ & $30 \cdot 3$ & $40 \cdot 3$ \\
\hline Technical/professional & $21 \cdot 2$ & $25 \cdot 8$ & $23 \cdot 0$ & $22 \cdot 6$ & $19 \cdot 3$ & $24 \cdot 6$ & $18 \cdot 7$ & $24 \cdot 8$ & $26 \cdot 0$ & $24 \cdot 0$ & $21 \cdot 4$ & $17 \cdot 1$ \\
\hline Secondary school & 14.9 & $7 \cdot 3$ & $11 \cdot 6$ & 14.5 & $10 \cdot 3$ & $9 \cdot 2$ & $19 \cdot 4$ & $19 \cdot 9$ & $16 \cdot 6$ & $19 \cdot 4$ & $19 \cdot 0$ & 20.9 \\
\hline Longer education & $20 \cdot 2$ & $21 \cdot 6$ & $17 \cdot 9$ & $20 \cdot 1$ & $26 \cdot 3$ & $17 \cdot 4$ & 23.0 & $16 \cdot 8$ & $15 \cdot 1$ & 20.5 & $21 \cdot 8$ & $16 \cdot 5$ \\
\hline Not specified & $6 \cdot 1$ & $2 \cdot 3$ & 1.5 & 4.6 & $2 \cdot 3$ & $3 \cdot 2$ & $11 \cdot 0$ & $3 \cdot 3$ & 4.5 & $7 \cdot 1$ & $7 \cdot 6$ & $5 \cdot 3$ \\
\hline \multicolumn{13}{|l|}{ Tumour site (\%) } \\
\hline Colon & $61 \cdot 5$ & $52 \cdot 6$ & $60 \cdot 4$ & $55 \cdot 6$ & $58 \cdot 3$ & $55 \cdot 2$ & $68 \cdot 5$ & $65 \cdot 5$ & $68 \cdot 2$ & $65 \cdot 0$ & $64 \cdot 3$ & $67 \cdot 7$ \\
\hline Rectum & 38.5 & $47 \cdot 4$ & $39 \cdot 7$ & $44 \cdot 4$ & $41 \cdot 8$ & $44 \cdot 8$ & 31.5 & 34.5 & $31 \cdot 8$ & $35 \cdot 0$ & 35.7 & $32 \cdot 3$ \\
\hline \multicolumn{13}{|l|}{ Tumour grade (\%) } \\
\hline Well differentiated & $4 \cdot 3$ & $4 \cdot 0$ & 3.5 & $3 \cdot 1$ & 3.5 & $6 \cdot 7$ & $9 \cdot 3$ & $12 \cdot 0$ & $8 \cdot 0$ & $9 \cdot 5$ & $10 \cdot 2$ & $12 \cdot 5$ \\
\hline Moderately differentiated & $25 \cdot 9$ & $21 \cdot 1$ & $26 \cdot 0$ & $25 \cdot 4$ & $26 \cdot 0$ & $24 \cdot 4$ & $30 \cdot 2$ & 34.5 & $30 \cdot 5$ & $29 \cdot 8$ & $29 \cdot 6$ & $34 \cdot 7$ \\
\hline Poorly/undifferentiated & $5 \cdot 5$ & $7 \cdot 3$ & $5 \cdot 8$ & $5 \cdot 8$ & $6 \cdot 8$ & $5 \cdot 2$ & 8.4 & $10 \cdot 2$ & 8.0 & 8.4 & 8.4 & 8.5 \\
\hline Unknown & $64 \cdot 2$ & $67 \cdot 7$ & $64 \cdot 7$ & 65.7 & $63 \cdot 8$ & 63.7 & $52 \cdot 1$ & $43 \cdot 3$ & $53 \cdot 5$ & $52 \cdot 3$ & 51.9 & $44 \cdot 3$ \\
\hline \multicolumn{13}{|l|}{ Tumour stage (\%) } \\
\hline Localised & $18 \cdot 6$ & $21 \cdot 8$ & $18 \cdot 7$ & $19 \cdot 0$ & $15 \cdot 3$ & $20 \cdot 7$ & $14 \cdot 2$ & $17 \cdot 0$ & $18 \cdot 5$ & $16 \cdot 6$ & $15 \cdot 6$ & $16 \cdot 2$ \\
\hline Localised with invasion & $13 \cdot 6$ & $18 \cdot 6$ & $16 \cdot 4$ & $18 \cdot 3$ & $17 \cdot 3$ & $15 \cdot 9$ & $16 \cdot 8$ & $19 \cdot 9$ & $14 \cdot 6$ & $18 \cdot 1$ & $14 \cdot 7$ & $19 \cdot 1$ \\
\hline Metastatic regional & $28 \cdot 2$ & $26 \cdot 6$ & $25 \cdot 3$ & $27 \cdot 4$ & $17 \cdot 5$ & $27 \cdot 9$ & 23.9 & $33 \cdot 6$ & $25 \cdot 8$ & $29 \cdot 8$ & $23 \cdot 4$ & $30 \cdot 9$ \\
\hline Metastatic distant & $7 \cdot 1$ & $11 \cdot 8$ & $10 \cdot 6$ & $13 \cdot 5$ & $8 \cdot 3$ & $8 \cdot 7$ & $8 \cdot 0$ & $11 \cdot 1$ & $11 \cdot 0$ & $10 \cdot 4$ & $8 \cdot 7$ & $9 \cdot 1$ \\
\hline Unknown & $32 \cdot 5$ & $21 \cdot 3$ & $29 \cdot 0$ & $21 \cdot 8$ & $41 \cdot 8$ & $26 \cdot 9$ & $37 \cdot 1$ & $18 \cdot 4$ & $30 \cdot 1$ & $25 \cdot 1$ & $37 \cdot 6$ & 24.9 \\
\hline
\end{tabular}

M, men; W, women; Q, quartile.

processed meat intake (47.4\%) compared with Q1 (38.5\%): the corresponding distribution among women was less varied (34.5 v. 31.5\% for Q4 and Q1 of red and processed meat intake, respectively).

In both the age-adjusted and multivariable analysis, there was no evidence of an association between pre-diagnostic intakes of red meat, poultry or fibre and death due to CRC (Table 2). For processed meat and CRC mortality, a marginally significant test for trend was detected across quartiles $(P=0.053$, Table 2$)$, but no association was detected among the individual quartiles or in the continuous analysis of grams per day. The corresponding results for all-cause mortality were also null, with the exception of a positive association in the highest quartile of processed meat that was limited to the age- and sex-adjusted model (hazard ratio (HR) 1.23; $95 \%$ CI 1.04, 1.46)) (Table 3).

Testing for interactions between the main dietary exposures (modelled as continuous variables) and other covariates yielded mostly null results (online Supplementary Table S1). A marginal interaction between poultry intake (continuous $\mathrm{g} / \mathrm{d}$ ) and sex was detected in relation to all-cause mortality ( $P$ value $0 \cdot 10)$; stratification by sex revealed a significant inverse association among women (HR/20 g 0.92; $95 \%$ CI 0.84, 1.00) but not among men (HR 1.00; 95\% CI 0.91, 1.09) (online Supplementary Table S2). For both CRC-specific and all-cause mortality, interactions were detected between processed meat intake $(30 \mathrm{~g} / \mathrm{d})$ and folate intake ( $P$ values 0.016 and 0.06 , respectively, online Supplementary Table S1); however, the associations for processed meat remained null when the sample was divided by tertiles of folate intake. For CRC-specific mortality, a marginal interaction was detected between poultry and BMI group ( $P$ value 0.06 ); stratification by BMI group yielded an inverse relationship among overweight adults $(\mathrm{HR} / 20 \mathrm{~g} 0.89 ; 95 \% \mathrm{CI}$ $0 \cdot 80,1 \cdot 00$ ) but no associations among normal-weight (HR 0.99; $95 \%$ CI $0 \cdot 87,1 \cdot 12$ ) or obese adults (HR 1.10; $95 \%$ CI 0.95, 1.27) (online Supplementary Table S1). There was no evidence of interactions by length of follow-up or lag-time between dietary assessment and CRC diagnosis. Owing to the large number of 
Table 2. Colorectal cancer (CRC)-specific mortality in relation to pre-diagnostic fibre and meat intakes among CRC survivors in European Prospective Investigation into Cancer and Nutrition (Hazard ratios (HR) and $95 \%$ confidence intervals)

\begin{tabular}{|c|c|c|c|c|c|c|c|c|c|c|c|}
\hline & \multicolumn{2}{|c|}{ Q1 } & \multicolumn{2}{|c|}{ Q2 } & \multicolumn{2}{|c|}{ Q3 } & \multicolumn{2}{|c|}{ Q4 } & \multirow[b]{2}{*}{$P_{\text {for trend }}$} & \multicolumn{2}{|c|}{ Continuous } \\
\hline & $\mathrm{HR}$ & $95 \% \mathrm{Cl}$ & $\mathrm{HR}$ & $95 \% \mathrm{Cl}$ & $\mathrm{HR}$ & $95 \% \mathrm{Cl}$ & $\mathrm{HR}$ & $95 \% \mathrm{Cl}$ & & $\mathrm{HR}$ & $95 \% \mathrm{Cl}$ \\
\hline Fibre (mean, g/d) & \multicolumn{2}{|c|}{14.5} & \multicolumn{2}{|c|}{$19 \cdot 5$} & \multicolumn{2}{|c|}{$24 \cdot 1$} & \multicolumn{2}{|c|}{$31 \cdot 2$} & & & \\
\hline Deaths $(n)$ & \multicolumn{2}{|c|}{259} & \multicolumn{2}{|c|}{249} & \multicolumn{2}{|c|}{265} & \multicolumn{2}{|c|}{235} & & \multicolumn{2}{|c|}{ Per $10 \mathrm{~g}$} \\
\hline Model $1^{*}$ & 1.0 & Ref. & 0.94 & $0.79,1.12$ & 0.99 & $0.83,1.18$ & 0.87 & $0.73,1.05$ & 0.15 & 0.97 & $0.90,1.06$ \\
\hline Model $2 \dagger$ & 1.0 & Ref. & 0.95 & $0.79,1.15$ & 1.04 & $0.85,1.27$ & 0.90 & $0.69,1.17$ & 0.13 & 1.00 & $0.87,1.15$ \\
\hline Red and processed meat (mean, $\mathrm{g} / \mathrm{d}$ ) & \multicolumn{2}{|c|}{$28 \cdot 6$} & \multicolumn{2}{|c|}{60.5} & \multicolumn{2}{|c|}{91.4} & \multirow{2}{*}{\multicolumn{2}{|c|}{141.4}} & & \multirow{2}{*}{\multicolumn{2}{|c|}{ Per $50 \mathrm{~g}$}} \\
\hline Deaths $(n)$ & \multicolumn{2}{|c|}{262} & \multicolumn{2}{|c|}{241} & \multicolumn{2}{|c|}{253} & & & & & \\
\hline Model $1^{\star}$ & 1.0 & Ref. & 0.89 & $0.75,1.07$ & 0.96 & $0.80,1.15$ & 1.00 & $0.83,1.20$ & 0.65 & 0.98 & $0.86,1.12$ \\
\hline Model $2 \dagger$ & 1.0 & Ref. & 0.90 & $0.75,1.08$ & 0.96 & $0.80,1 \cdot 16$ & 1.00 & $0.81,1.23$ & 0.90 & 0.99 & $0.84,1 \cdot 15$ \\
\hline Red meat (mean, g/d) & \multicolumn{2}{|c|}{9.6} & \multicolumn{2}{|c|}{29.5} & \multirow{2}{*}{\multicolumn{2}{|c|}{$\begin{array}{c}53 \cdot 1 \\
238\end{array}$}} & \multirow{2}{*}{\multicolumn{2}{|c|}{$\begin{array}{l}91.0 \\
263\end{array}$}} & & \multirow{2}{*}{\multicolumn{2}{|c|}{ Per $40 \mathrm{~g}$}} \\
\hline Deaths $(n)$ & \multicolumn{2}{|c|}{262} & \multicolumn{2}{|c|}{245} & & & & & & & \\
\hline Model $1^{*}$ & 1.0 & Ref. & 1.00 & $0.84,1.20$ & 0.92 & $0.76,1 \cdot 11$ & 0.92 & $0.75,1 \cdot 13$ & 0.30 & 0.97 & $0.88,1.06$ \\
\hline Model $2 \dagger$ & 1.0 & Ref. & 0.91 & $0.76,1 \cdot 10$ & 0.87 & $0.72,1.06$ & 0.93 & $0.75,1.15$ & 0.46 & 0.99 & $0.89,1.10$ \\
\hline Processed meat (mean, $\mathrm{g} / \mathrm{d}$ ) & & 9 & & & & 5.3 & & & & & \\
\hline Deaths $(n)$ & & & & & & & & & & & $30 \mathrm{~g}$ \\
\hline Model $1^{\star}$ & 1.0 & Ref. & 1.04 & $0.87,1.25$ & 1.13 & $0.94,1.36$ & $1 \cdot 17$ & $0.94,1.42$ & 0.030 & 1.01 & $0.97,1.06$ \\
\hline Model $2 \dagger$ & 1.0 & Ref. & 0.95 & $0.79,1.15$ & 1.07 & $0.88,1.30$ & $1 \cdot 12$ & $0.90,1.39$ & 0.053 & 1.00 & $0.95,1.05$ \\
\hline Poultry (mean, g/d) & & 6 & & 3 & & 3 & & & & & \\
\hline Deaths $(n)$ & & & & & & & & & & & $20 \mathrm{~g}$ \\
\hline Model $1^{*}$ & 1.0 & Ref. & 1.00 & $0.84,1.20$ & 1.12 & $0.94,1.35$ & 0.93 & $0.77,1.13$ & 0.31 & 0.96 & $0.90,1.03$ \\
\hline Model $2 \dagger$ & 1.0 & Ref. & 0.97 & $0.81,1.16$ & $1 \cdot 13$ & $0.94,1.36$ & 0.91 & $0.75,1 \cdot 10$ & 0.17 & 0.96 & $0.89,1.03$ \\
\hline
\end{tabular}

Q, quartile; Ref., referent values; $M$, men; W, women.

* Model 1: adjusted for age at diagnosis (1-year increments) and sex; stratified by country.

† Model 2: adjusted for age at diagnosis (1-year increments), sex, BMI (continuous), smoking status (current, former, never, unknown), tumour grade (well differentiated, moderately differentiated, poorly/undifferentiated, unknown), tumour stage (I, II, III, IV, unknown), year of tumour diagnosis (continuous), energy intake (kJ/d (kcal/d)), Ca intake (mg/d), folate intake (mg/d), alcohol intake ( $/ \mathrm{g} / \mathrm{d}: 0,>0-6(\mathrm{M}) />0-3(\mathrm{~W}),>6-12(\mathrm{M}) />3-12(\mathrm{~W}),>12-24,>24-60,>60)$ and ( 
Table 3. All-cause mortality in relation to pre-diagnostic fibre and meat intakes among colorectal cancer survivors in European Prospective Investigation into Cancer and Nutrition

(Hazard ratios (HR) and $95 \%$ confidence intervals)

\begin{tabular}{|c|c|c|c|c|c|c|c|c|c|c|c|}
\hline & \multicolumn{2}{|c|}{ Q1 } & \multicolumn{2}{|c|}{ Q2 } & \multicolumn{2}{|c|}{ Q3 } & \multicolumn{2}{|c|}{ Q4 } & \multirow[b]{2}{*}{$P_{\text {for trend }}$} & \multicolumn{2}{|c|}{ Continuous } \\
\hline & $\mathrm{HR}$ & $95 \% \mathrm{Cl}$ & $\mathrm{HR}$ & $95 \% \mathrm{Cl}$ & $\mathrm{HR}$ & $95 \% \mathrm{Cl}$ & $\mathrm{HR}$ & $95 \% \mathrm{Cl}$ & & $\mathrm{HR}$ & $95 \% \mathrm{Cl}$ \\
\hline \multicolumn{12}{|l|}{ Fibre } \\
\hline Deaths $(n)$ & \multicolumn{2}{|c|}{316} & \multicolumn{2}{|c|}{323} & \multicolumn{2}{|c|}{320} & \multicolumn{2}{|c|}{302} & & \multicolumn{2}{|c|}{ Per $10 \mathrm{~g}$} \\
\hline Model $1^{*}$ & 1.0 & Ref. & 0.98 & $0.83,1.14$ & 0.95 & $0.81,1.11$ & 0.89 & $0.76,1.05$ & 0.20 & 0.98 & $0.91,1.06$ \\
\hline Model 2† & 1.0 & Ref. & 0.96 & $0.81,1.14$ & 0.93 & $0.77,1.12$ & 0.84 & $0.66,1.06$ & 0.57 & 0.95 & $0.84,1.08$ \\
\hline \multicolumn{12}{|c|}{ Red and processed meat } \\
\hline Deaths $(n)$ & \multicolumn{2}{|c|}{331} & \multicolumn{2}{|c|}{300} & \multicolumn{2}{|c|}{309} & \multicolumn{2}{|c|}{321} & & \multicolumn{2}{|c|}{ Per $50 \mathrm{~g}$} \\
\hline Model $1^{*}$ & 1.0 & Ref. & 0.92 & $0.78,1.03$ & 0.96 & $0.82,1.13$ & 1.04 & $0.88,1.22$ & 0.99 & 1.02 & $0.91,1.15$ \\
\hline Model 2† & 1.0 & Ref. & 0.91 & $0.77,1.07$ & 0.94 & $0 \cdot 79,1 \cdot 11$ & 1.00 & $0.83,1.20$ & 0.38 & 1.01 & $0.88,1.16$ \\
\hline \multicolumn{12}{|l|}{ Red meat } \\
\hline Deaths $(n)$ & \multicolumn{2}{|c|}{319} & \multicolumn{2}{|c|}{319} & \multicolumn{2}{|c|}{293} & \multicolumn{2}{|c|}{330} & & \multicolumn{2}{|c|}{ Per $40 \mathrm{~g}$} \\
\hline Model $1^{\star}$ & 1.0 & Ref. & 1.01 & $0.86,1 \cdot 18$ & 0.85 & $0.72,1.01$ & 0.94 & $0.79,1.13$ & 0.22 & 0.97 & $0.90,1.06$ \\
\hline Model $2 \dagger$ & 1.0 & Ref. & 1.01 & $0.86,1 \cdot 18$ & 0.87 & $0.73,1.04$ & 0.95 & $0.78,1.14$ & 0.47 & 0.98 & $0.90,1.07$ \\
\hline \multicolumn{12}{|c|}{ Processed meat } \\
\hline Deaths $(n)$ & \multicolumn{2}{|c|}{326} & \multicolumn{2}{|c|}{316} & \multicolumn{2}{|c|}{306} & \multicolumn{2}{|c|}{313} & & \multicolumn{2}{|c|}{ Per $30 \mathrm{~g}$} \\
\hline Model $1^{\star}$ & 1.0 & Ref. & 1.05 & $0.90,1 \cdot 24$ & $1 \cdot 10$ & $0.93,1.29$ & 1.23 & $1.04,1.46$ & 0.14 & 1.03 & $0.99,1.07$ \\
\hline Model 2† & 1.0 & Ref. & 0.98 & $0.83,1.15$ & 1.04 & $0.88,1.24$ & $1 \cdot 17$ & $0.97,1.42$ & 0.20 & 1.02 & $0.98,1.07$ \\
\hline \multicolumn{12}{|l|}{ Poultry } \\
\hline Deaths $(n)$ & \multicolumn{2}{|c|}{330} & & 322 & & 03 & & 06 & & & $20 \mathrm{~g}$ \\
\hline Model $1^{*}$ & 1.0 & Ref. & 0.95 & $0.81,1.11$ & 1.02 & $0.86,1.20$ & 0.91 & $0.77,1.07$ & 0.44 & 0.96 & $0.91,1.02$ \\
\hline Model $2 \dagger$ & 1.0 & Ref. & 0.91 & $0.77,1.07$ & 0.99 & $0.83,1 \cdot 16$ & 0.87 & $0.73,1.03$ & 0.35 & 0.96 & $0.90,1.02$ \\
\hline
\end{tabular}

$\mathrm{Q}$, quartile; Ref., referent values; M, men; W, women.

* Model 1: adjusted for age at diagnosis (1-year increments) and sex; stratified by country.

† Model 2: adjusted for age at diagnosis (1-year increments), sex, BMI (continuous), smoking status (current, former, never, unknown), tumour grade (well differentiated, moderately differentiated, poorly/undifferentiated, unknown), tumour stage (I, II, III, IV, unknown), year of tumour diagnosis (continuous), energy intake (kJ/d (kcal/d)), Ca intake (mg/d), folate intake (mg/d), alcohol intake (g/d: 0, >0-6 (M)/>0-3 (W), >6-12 (M)/>3-12 (W), >12-24, >24-60, >60), and education (primary school, technical/professional school, secondary school, longer education (including university, unknown)); stratified by country.

interactions tested, significant or marginally significant results may have been due to chance.

Further adjustment of the multivariable models to include waist circumference did not alter the null associations detected, nor did mutual adjustment for red meat, processed meat, poultry and fibre intakes. The effect of substituting poultry for red and processed meat was not significant for all-cause mortality (HR/20 g $0.95 ; 95 \%$ CI $0.87,1.03)$ or CRC-specific death $(\mathrm{HR} / 20 \mathrm{~g} 0.96 ; 95 \% \mathrm{CI} 0.88,1.06)$ (data not shown in tables). Fibre from cereal, vegetable or fruit sources was not associated with either CRC-specific or all-cause mortality in multivariable models (online Supplementary Table S3 and S4); these results remained null in further models where all fibre sources were examined simultaneously (data not shown). Similarly, results were not altered by the exclusion of deaths that occurred within 6 months of CRC diagnosis, limiting the analysis to those with stage I, II or III tumours or exploring the influence of missing stage data as described in the methods section (data not shown).

\section{Discussion}

In the present analysis, pre-diagnostic intake of red meat or fibre was not associated with survival after CRC diagnosis. Suggestive evidence was found for an inverse association between poultry intake and mortality among women and for a positive association between processed meat intake and risk of CRC-specific mortality. The associations were unchanged throughout a variety of sensitivity analyses and missing data exploration. Overall, these results are in contrast to the relatively consistent evidence regarding CRC prevention, where higher fibre and lower red or processed meat intakes are associated with lower CRC risk ${ }^{(11)}$. However, the number of studies available on meat and fibre intakes and CRC survival is still considerably smaller than that for CRC incidence; therefore, further research is required to determine whether dietary recommendations for CRC prevention can be extended to CRC survival.

The results of the present study can be compared with the available evidence for meat and fibre as individual exposures and for dietary patterns that feature meat and fibre as predominant components. The null result for fibre intake and CRC survival in the present study is consistent with several earlier studies ${ }^{(20,21,30)}$. The predominantly Mormon population among whom higher pre-diagnostic fibre intake was associated with poorer CRC survival has unique characteristics (prohibited from alcohol, caffeine and tobacco), which render it less comparable with the EPIC population ${ }^{(19)}$. For meat intake, a positive association in previous studies was limited to subgroups that we were not able to examine within EPIC: those with a first-degree relative with $\mathrm{CRC}^{(18)}$ (post-diagnostic intake) and those who had meat intakes consistently above the median before and after diagnosis ${ }^{(17)}$. In observational studies of postdiagnostic dietary patterns, a Western pattern characterised by high intake of red and processed meat was found to be associated with poorer outcomes among CRC survivors ${ }^{(31-33)}$. The results for post-diagnostic dietary patterns are not entirely consistent: one study found that the Alternative Healthy Eating 
Index (A-HEI)-2010 was associated with lower mortality, but found no association for the DASH (Dietary Approaches to Stop Hypertension), Alternate Med Score, 'Western' pattern or 'Prudent' pattern ${ }^{(31)}$. Among the individual components of the A-HEI, a significant association with all-cause mortality was found only for greater post-diagnostic consumption of sugarsweetened beverages $(\mathrm{SSB})^{(31)}$. SSB also contributed to the data-derived patterns associated with poorer CRC survival outcomes in studies from Canada ${ }^{(33)}$ and the $\mathrm{USA}^{(32)}$. In an earlier analysis of CRC survival in EPIC, SSB were included in an index based on pre-diagnostic adherence to World Cancer Research Fund/American Institute for Cancer Research (WCRF/ AICR) cancer prevention recommendations; greater adherence to the recommendations was associated with improved CRC survival ${ }^{(34)}$. However, exclusion of SSB from the index did not attenuate the protective effect of higher WCRF/AICR scores among CRC survivors ${ }^{(34)}$, and thus it is unlikely that the null results of the present analysis can be explained by a failure to account for SSB intake. In contrast to the present results, an inverse association was detected between the pre-diagnostic plant product component of the WCRF/AICR score and CRCspecific mortality; however, for the animal products component, results were similarly non-significant ${ }^{(34)}$. Overall, the interpretation of index components remains complex; in one study, the pre-diagnostic red and processed meat pattern associated with poorer CRC survival included fish as a component ${ }^{(33)}$, which has previously been associated with lower risk of CRC incidence ${ }^{(35)}$.

It is thought that cancer recurrence can be partly attributed to proliferation of micrometastatic disease that was not removed at the time of surgery. Accordingly, it is possible that some dietary factors associated with the development and progression of incident CRC tumours may also be associated with recurrence; one of the mechanisms by which diet could be related to CRC survival outcomes is through modification of circulating insulin levels, which is positively associated with greater production of insulin-like growth factor (IGF)- ${ }^{(36)}$. For example, 'prudent' dietary patterns (characterised by higher intake of fruits, vegetables, poultry, fish, whole grains and legumes) have been associated with lower levels of insulin, whereas 'Western' dietary patterns (characterised by higher intakes of red meat, processed meat, French fries, eggs, high-fat dairy products, sweets and refined grains) were positively associated with insulin and C-peptide levels ${ }^{(37)}$. Evidence from animal models indicates that greater IGF-1 exposure is associated with an increased rate of tumour progression ${ }^{(38)}$. In human studies, the results are more complex: there was no association between post-diagnostic IGF-1 levels and overall survival, but post-diagnostic insulin-like growth factor binding protein 3 (IGFBP-3) was inversely associated with tumour progression $^{(39)}$. In addition, a diet high in fibre can result in higher levels of SCFA in the lumen, which can serve to induce apoptosis in tumour cells ${ }^{(40)}$.

Despite these potential pathways, we found no association between pre-diagnostic red meat or fibre intakes and CRC survival, and only marginally suggestive results for processed meat. An implicit assumption of the present analysis is that prediagnostic dietary intake is predictive of post-diagnostic diet. It is plausible that for some participants surgical treatment of CRC results in complications that require temporary or permanent dietary adjustments to avoid intestinal discomfort. A study on CRC survivors reported that, although the majority of respondents resumed comfort with their diet within 12 months, avoidance of foods such as fruits and vegetables was higher among those with a permanent ostomy (surgically created opening in the body for the discharge of body waste, $9.1 \%) v$. those who had their ostomy reversed $(3.6 \%)^{(41)}$. In contrast, a comparison of repeat dietary assessments in a cohort of Norwegian women yielded no differences in consumption of fibre, red meat or poultry between the pre-and post-diagnostic period among CRC survivors ${ }^{(42)}$. Intake of total meat products was lower during the post-diagnostic dietary assessment among the Norwegian CRC survivors, but the change was comparable with that observed in cancer-free women in the cohort ${ }^{(42)}$. In principle, studies with the greatest potential to influence the development of cancer survival recommendations would be those with multiple dietary assessments both before and after diagnosis. Some of the aforementioned limitations may be addressed in the future by a multistage CRC survival-specific cohort that is underway at present ${ }^{(43)}$. In the interim, the present analysis contributes to limited literature on diet and CRC survival.

The design of the present study had a number of strengths in terms of capacity to examine CRC survival. The collection of pre-diagnostic dietary data avoided the issue dietary variability close to the time of diagnosis, and thus avoided reverse causality, and standardised dietary assessment was undertaken. A range of sensitivity analyses was undertaken, including the exclusion of early mortality events, advanced stage tumours and stratification by duration since dietary assessment. The sample size was large, and there were data on a wide range of potentially confounding covariates including general and abdominal obesity (BMI and waist circumference), Ca, folate and alcohol intakes, and smoking status.

In conclusion, we found no evidence of an association between pre-diagnostic intakes of red meat or fibre and CRC survival. There is suggestive evidence of an association between poultry intake and all-cause mortality among female CRC survivors, and between processed meat intake and CRCspecific mortality; however, further research using postdiagnostic dietary data is required to confirm this relationship.

\section{Acknowledgements}

The coordination of EPIC is financially supported by the European Commission (Directorate General for Health and Consumer Affairs; DG-SANCO) and the International Agency for Research on Cancer. The national cohorts are supported by Danish Cancer Society (Denmark); Ligue Contre le Cancer, Institut Gustave Roussy, Mutuelle Générale de l'Education Nationale and Institut National de la Santé et de la Recherche Médicale (France); Deutsche Krebshilfe, Deutsches Krebsforschungszentrum and Federal Ministry of Education and Research (Germany); the Hellenic Health Foundation (Greece); Associazione Italiana per la Ricerca sul Cancro (AIRC) and National Research Council (Italy); Dutch Ministry of Public 
Health, Welfare and Sports (VWS), Netherlands Cancer Registry (NKR), LK Research Funds, Dutch Prevention Funds, Dutch ZON (Zorg Onderzoek Nederland), World Cancer Research Fund and Statistics Netherlands (The Netherlands); Nordic Center of Excellence programme on Food, Nutrition and Health (Norway); Health Research Fund (FIS), PI13/00061 to Granada), Regional Governments of Andalucía, Asturias, Basque Country, Murcia (no. 6236) and Navarra and ISCIII RETIC (Instituto de Salud Carlos III Las Redes temáticas de investigación cooperativa en salud) (RD06/0020) (Spain); World Cancer Research Foundation ref. 2011/428, AGAUR (Agency for Management of University and Research Grants), Generalitat de Catalunya (exp. 2014 SGR 726), Health Research Funds (FIS Exp PI11/1486) and RD12/0036/0018 and Catalan Institute of Oncology (ICO-IDIBELL)(Barcelona); Swedish Cancer Society, Swedish Scientific Council and County Councils of Skåne and Västerbotten (Sweden); Cancer Research UK (14136 to EPIC-Norfolk; C570/ A16491 to EPIC-Oxford) and Medical Research Council (1000143 to EPIC-Norfolk) (UK).

H. A. W. and T. N. conceived the idea, drafted the manuscript, had full access to all the data in the study and take responsibility for the integrity of the data and the accuracy of the data analysis. H. A. W. performed the statistical analyses. H. A. W., T. N., K. O., C. C. D., H. B. B.-d.-M., V. F., M. J., F. J. B. v. D., G. S., D. R.-B. and N. M. interpreted the results. K. O., H. B. B.-d.-M., M. J., F. J. B. v. D., G. S., A. Tj., A. O., M.-C. B.-R., V. K., T. K., K. A., H. B., A. Tr., P. L., C. B., D. P., S. S., R. T., P. H. P., E. W., P. J., J. R. Q., M.-J. S., M. D., J.-M. H., A. B., I. J., K. E. B., K.-T. K., N. J. W., A. J. C. and E. R. are EPIC investigators and were responsible for the study design and data collection. All authors critically revised the manuscript for intellectual content and approved the final version of the manuscript.

Data sharing: for information on how to submit an application for gaining access to EPIC data and/or biospecimens, please follow the instructions at http://epic.iarc.fr/access/ index.php

The authors declare that there are no conflicts of interest

\section{Supplementary material}

For supplementary material/s referred to in this article, please visit http://dx.doi.org/doi:10.1017/S0007114516001859

\section{References}

1. Cancer Research UK (2015) Statistics on preventable cancers: preventable cancer cases by cancer type. Online resource. http://www.cancerresearchuk.org/health-professional/cancerstatistics/risk/preventable-cancers\#heading-One (accessed October 2015).

2. Cross AJ, Pollock JRA \& Bingham SA (2003) Haem, not protein or inorganic iron, is responsible for endogenous intestinal $\mathrm{N}$-nitrosation arising from red meat. Cancer Res $\mathbf{6 3}$, 2358-2360.

3. Fung KYC, Cosgrove L, Lockett T, et al. (2012) A review of the potential mechanisms for the lowering of colorectal oncogenesis by butyrate. BrJ Nutr 108, 820-831.

4. Pufulete M, Al-Ghnaniem R, Khushal A, et al. (2005) Effect of folic acid supplementation on genomic DNA methylation in patients with colorectal adenoma. Gut 54, 648-653.
5. Bostick RM, Fosdick L, Wood JR, et al. (1995) Calcium and colorectal epithelial-cell proliferation in sporadic adenoma patients - a randomized, double-blinded, placebo-controlled clinical-trial. J Natl Cancer Inst 87, 1307-1315.

6. Hamer HM, Jonkers D, Venema K, et al. (2008) Review article: the role of butyrate on colonic function. Aliment Pharmacol Ther 27, 104-119.

7. Newmark HL, Wargovich MJ \& Bruce WR (1984) Colon cancer and dietary-fat, phosphate, and calcium - a hypothesis. J Natl Cancer Inst 72, 1323-1325.

8. Burkitt DP (1971) Epidemiology of cancer of colon and rectum. Cancer 28, 3-13.

9. Pereira MA, O'Reilly E, Augustsson K, et al. (2004) Dietary fiber and risk of coronary heart disease: a pooled analysis of cohort studies. Arch Intern Med 164, 370-376.

10. Calle EE \& Kaaks R (2004) Overweight, obesity and cancer: epidemiological evidence and proposed mechanisms. Nat Rev Cancer 4, 579-591.

11. World Cancer Research Fund \& American Institute for Cancer Research (2011) Continuous Update Project report. Food, nutrition, physical activity, and the prevention of colorectal cancer. http://www.wcrf.org/sites/default/files/ColorectalCancer-2011-Report.pdf

12. Pullar JM, Chisholm A \& Jackson C (2012) Dietary information for colorectal cancer survivors: an unmet need. $N Z$ Med J $\mathbf{1 2 5}$, $27-37$.

13. World Cancer Research Fund \& American Institute for Cancer Research (2007) Food, Nutrition, Physical Activity, and the Prevention of Cancer: A Global Perspective. Washington, DC: AIRC.

14. Edwards BK, Noone AM, Mariotto AB, et al. (2014) Annual report to the nation on the status of cancer, 1975-2010, featuring prevalence of comorbidity and impact on survival among persons with lung, colorectal, breast, or prostate cancer. Cancer 120, 1290-1314.

15. Quaresma M, Coleman MP \& Rachet B (2014) 40-year trends in an index of survival for all cancers combined and survival adjusted for age and sex for each cancer in England and Wales, 1971-2011: a population-based study. Lancet $\mathbf{3 8 5}$, 1206-1218.

16. Ouakrim DA, Pizot C, Boniol M, et al. (2015) Trends in colorectal cancer mortality in Europe: retrospective analysis of the WHO mortality database. BMJ 351, h4970.

17. McCullough ML, Gapstur SM, Shah R, et al. (2013) Association between red and processed meat intake and mortality among colorectal cancer survivors. J Clin Oncol 31, 2773-2782.

18. Zell JA, Ignatenko NA, Yerushalmi HF, et al. (2006) Risk and risk reduction involving arginine intake and meat consumption in colorectal tumorigenesis and survival. Int $J$ Cancer 120, 459-468.

19. Slattery ML, French TK, Egger MJ, et al. (1989) Diet and survival of patients with colon cancer in Utah: is there an association? Int J Epidemiol 18, 792-797.

20. Dray X, Boutron-Ruault MC, Bertrais S, et al. (2003) Influence of dietary factors on colorectal cancer survival. Gut $\mathbf{5 2}$, 868-873.

21. Skeie G, Braaten T, Olsen A, et al. (2014) Whole grain intake and survival among Scandinavian colorectal cancer patients. Nutr Cancer 66, 6-13.

22. Riboli E \& Kaaks R (1997) The EPIC project: rationale and study design. Int J Epidemiol 26, S6-S14.

23. Riboli E, Hunt KJ, Slimani N, et al. (2002) European Prospective Investigation into Cancer and Nutrition (EPIC): study populations and data collection. Public Health Nutr $\mathbf{5}$, $1113-1124$. 
24. Slimani N, Deharveng G, Unwin I, et al. (2007) The EPIC nutrient database project (ENDB): a first attempt to standardize nutrient databases across the 10 European countries participating in the EPIC study. Eur J Clin Nutr $\mathbf{6 1}$, 1037-1056.

25. Spencer EA, Appleby PN, Davey GK, et al. (2002) Validity of self-reported height and weight in 4808 EPIC-Oxford participants. Public Health Nutr 5, 561-565.

26. Fedirko V, Riboli E, Tjonneland A, et al. (2012) Prediagnostic 25-hydroxyvitamin D, VDR and CASR polymorphisms, and survival in patients with colorectal cancer in western European populations. Cancer Epidemiol Biomarkers Prev 21, 582-593.

27. Kohl M, Plischke M, Leffondre K, et al. (2015) PSHREG: a SAS macro for proportional and nonproportional subdistribution hazards regression. Comput Methods Programs Biomed 118, 218-233.

28. Wareham NJ, Jakes RW, Rennie KL, et al. (2003) Validity and repeatability of a simple index derived from the short physical activity questionnaire used in the European Prospective Investigation into Cancer and Nutrition (EPIC) study. Public Health Nutr 6, 407-413.

29. Kipnis V, Freedman LS, Brown CC, et al. (1993) Interpretation of energy adjustment models for nutritional epidemiology. Am J Epidemiol 137, 1376-1380.

30. Zell JA, McEligot AJ, Ziogas A, et al. (2007) Differential effects of wine consumption on colorectal cancer outcomes based on family history of the disease. Nutr Cancer 59, $36-45$.

31. Fung TT, Kashambwa R, Sato K, et al. (2014) Post diagnosis diet quality and colorectal cancer survival in women. PLOS ONE 9, e115377.

32. Meyerhardt JA, Niedzwiecki D, Hollis D, et al. (2007) Association of dietary patterns with cancer recurrence and survival in patients with stage III colon cancer. JAMA 298, 754-764.

33. Zhu Y, Wu H, Wang PP, et al. (2013) Dietary patterns and colorectal cancer recurrence and survival: a cohort study. BMJ Open 3, e002270.
34. Romaguera D, Ward H, Wark PA, et al. (2015) Pre-diagnostic concordance with the WCRF/AICR guidelines and survival in European colorectal cancer patients: a cohort study. BMC Med 13, 107.

35. Norat T, Bingham S, Ferrari P, et al. (2005) Meat, fish, and colorectal cancer risk: the European Prospective Investigation into Cancer and Nutrition. J Natl Cancer Inst 97, 906-916.

36. LeRoith D \& Roberts CT (2003) The insulin-like growth factor system and cancer. Cancer Lett 195, 127-137.

37. Fung TT, Rimm EB, Spiegelman D, et al. (2001) Association between dietary patterns and plasma biomarkers of obesity and cardiovascular disease risk. Am J Clin Nutr 73, 61-67.

38. Wu YP, Yakar S, Zhao L, et al. (2002) Circulating insulin-like growth factor-I levels regulate colon cancer growth and metastasis. Cancer Res 62, 1030-1035.

39. Fuchs CS, Goldberg RM, Sargent DJ, et al. (2008) Plasma insulin-like growth factors, insulin-like binding protein-3, and outcome in metastatic colorectal cancer: results from intergroup Trial N9741. Clin Cancer Res 14, 8263-8269.

40. Hague A, Elder DJE, Hicks DJ, et al. (1995) Apoptosis in colorectal tumor-cells - induction by the short-chain fatty-acids butyrate, propionate and acetate and by the bile-salt deoxycholate. Int J Cancer 60, 400-406.

41. Sun V, Grant M, Wendel CS, et al. (2015) Dietary and behavioral adjustments to manage bowel dysfunction after surgery in long-term colorectal cancer survivors. Ann Surg Oncol 22, 4317-4324.

42. Skeie G, Hjartaker A, Braaten T, et al. (2009) Dietary change among breast and colorectal cancer survivors and cancer-free women in the Norwegian women and cancer cohort study. Cancer Causes Control 20, 1955-1966.

43. Winkels RM, Heine-Broring RC, van ZM, et al. (2014) The COLON study: Colorectal cancer: Longitudinal, Observational study on Nutritional and lifestyle factors that may influence colorectal tumour recurrence, survival and quality of life. $B M C$ Cancer 14, 374 